\title{
ESTIMACIÓN DE PARÁMETROS GENÉTICOS EN CUYES UTILIZANDO ASReml-R
}

\author{
GENETICS PARAMETERS ESTIMATION IN GUINEA PIGS USING ASReml-R
}

\author{
${ }^{1}$ Ana Vargas
}

\begin{abstract}
Resumen
El objetivo de este estudio fue estimar parámetros genéticos para el peso al nacimiento y peso al destete en cuyes (Cavia porcellus) de Cieneguilla a través de un modelo lineal mixto que toma como estructura de correlación entre los efectos del componente aleatorio la estructura de parentesco entre los individuos. Como factores explicativos se consideró sexo de la cría, número de parto, estación, animal y camada; además para la respuesta peso al nacimiento se incluyó también como factores el total de nacidos y para la respuesta peso al destete se incluyó el número de nacidos vivos y edad de la cría. Se estimó la heredabilidad utilizando REML con el algoritmo AI implementado en ASReml-R. Los valores estimados de la heredabilidad para el peso al nacimiento fue de $0.158 \pm 0.036 \mathrm{y}$ para el peso al destete fue de $0.24 \pm 0.05$
\end{abstract}

Palabras clave: heredabilidad, mixed model, REML, ASReml-R.

\begin{abstract}
The objective was to estimate genetic parameters for birth and weaning weight in Cieneguilla guinea pigs (Cavia porcellus). A mixed model with pedigree information in the correlation structure between random effects was used. That model included sex of calf, parity, season of birth, animal and litter effect was used in the genetic analyses. In addition, the effect of number of newborns was included in the model for birth weight, the number of born alive and age at weaning were included in the model for weaning weight. Heritability was estimated by REML sampling with AI algorithm by using ASReml-R. Heritability estimates for birth weight was $0.158 \pm 0.036$ and for weaning weight was $0.24 \pm 0.05$.
\end{abstract}

Key words: heritability, mixed model, REML, ASReml-R.

\section{Introdución}

La estimación de parámetros genéticos como la heredabilidad es de gran importancia en el mejoramiento genético animal porque permite predecir el valor de una característica de interés (deseable a ser mejorada) que tendría la descendencia de cada uno de los animales observados y que por tanto llevaría a una mejor decisión en la selección de individuos que darían origen a las próximas generaciones. Todo esto se realiza con la finalidad de incrementar la característica de interés y así aumentar el rendimiento en la producción.

Para realizar estas estimaciones uno de los procedimientos estadísticos ampliamente utilizados se basa en máxima verosimilitud restringida conocida como REML (Thompson, 2008). La idea básica de estimación REML es estimar los componentes de varianza sobre los residuales calculados después de ajustar por mínimos cuadrados la parte de los efectos fijos del modelo. En el plano computacional se han implementado varios softwares tanto comerciales como libres tales como WOMBAT, ASReml, BGF90, DFREML, PEST/VCE, etc, que permiten ajustar un modelo mixto con información del parentesco de los individuos (pedigree) desde la perspectiva de genética cuantitativa. Por otro lado el lenguaje estadístico R, se ha convertido en uno de los lenguajes de desarrollo más utilizados en la actualidad que no sólo es desarrollado por estadísticos, sino por investigadores de distintas áreas a nivel internacional. Además, por ser un lenguaje "open source" (código abierto), ha permitido el trabajo colaborativo entre investigadores proporcionando una vasta contribución a través de sus paquetes (librerías) cuya documentación proporcionada es bastante detallada. ASReml-R es la interface de ASReml (Gilmour et al., 1999) en R, diseñado para ajustar modelos mixtos complejos (desbalanceados, muestras grandes, etc) a partir de al algoritmo AI (Average Information) utilizando métodos más eficientes de matriz sparse.

Este trabajo tuvo por objetivo estimar la heredabilidad del peso al nacer y peso al destete en cuyes de Cieneguilla a partir de modelos mixtos que incluyen información del parentesco utilizando ASREML-R.

\section{Materiales y métodos}

El modelo animal es un modelo mixto expresado como:

$$
\mathbf{y}=\mathbf{X} \boldsymbol{\beta}+\mathrm{Zu}+\mathbf{e}
$$


Donde $\mathbf{y}$ representa al vector de observaciones de valores fenotípicos, $\boldsymbol{\beta}$ y $\mathbf{u}$ son vectores desconocidos para efectos fijos y aleatorios respectivamente, $\mathbf{X}$ y $\mathbf{Z}$ son matrices diseños y e son los errores de los residuales. Asumiendo normalidad para la variable respuesta se tiene

$$
\begin{aligned}
& \left(\begin{array}{l}
\mathbf{y} \\
\mathbf{u} \\
\mathbf{e}
\end{array}\right)=N\left(\left(\begin{array}{c}
\mathbf{X} \boldsymbol{\beta} \\
\mathbf{0} \\
\mathbf{0}
\end{array}\right),\left(\begin{array}{ccc}
\mathbf{V} & \mathrm{ZG} & \mathbf{R} \\
\mathbf{G Z} & \mathbf{G} & 0 \\
\mathbf{R} & 0 & \mathbf{G}
\end{array}\right)\right) \\
& \mathrm{V}=\mathrm{ZGZ}^{\prime}+\mathbf{R}
\end{aligned}
$$

El aspecto genético viene dado por la especificación de la matriz de la covarianza entre los elementos $\mathbf{u}$, los cuales generalmente representan la suma de los efectos genéticos aditivos de los individuos en el pedigree. Para un modelo con una variable respuesta la matriz de covarianza de efectos aditivos es expresada como $\mathbf{G}=\mathbf{A} \sigma^{2}$, donde $\mathbf{A}$ es una matriz de coeficientes de las relaciones aditivas (Gutiérrez, J.P. 2010) y es calculada a través de la información de parentesco entre los individuos.

Estimar $\boldsymbol{\beta}$ y u matemáticamente llevó a Henderson (1950) a las siguientes ecuaciones simultáneas llamadas ecuaciones de modelos mixtos (MME), según Mrode (2014):

\section{$\left(\begin{array}{cc}\mathrm{XR}^{-1} \mathrm{X} & \mathrm{XR}^{-1} \mathrm{Z} \\ \mathrm{XR}^{-1} \mathrm{X} & \mathrm{ZR}^{-1} \mathrm{Z}+\mathrm{G}^{-1}\end{array}\right)\left(\begin{array}{l}\hat{\beta} \\ \hat{\mathbf{u}}\end{array}\right)=\left(\begin{array}{l}\mathrm{XR}^{-1} \mathrm{y} \\ \mathrm{ZR}^{-1} \mathrm{y}\end{array}\right)$}

Para estimar componentes de varianza a partir de un modelo animal, se genera ecuaciones del modelo mixto (MME) con matrices sparse (con muchos ceros), por lo que el procedimiento computacional es bastante complejo ya que involucra almacenamiento, factorización e inversión de matrices sparse (con muchos ceros). El algoritmo AI proporciona los errores estándares de los estimadores asintóticamente.

Para la estimación de los parámetros genéticos se utilizó la información sobre los pesos al nacer y al destete de 5120 crías en 1704 camadas, 117 progenitores machos y 868 progenitores hembras de la granja de cuyes de Cieneguilla de la Universidad Nacional Agraria La Molina, recolectados de cuyes mejorados entre enero 2009 y julio 2011. El destete se realizó a los 14 días de edad en promedio. La alimentación fue en base a los residuos de cosecha de brócoli y alimentos balanceados elaborados en la granja.

La estimación de componentes de varianza se llevó a cabo utilizando máxima verosimimilitud restringida, REML, aplicando el algoritmo AI implementado en ASReml-R, los modelos utilizados se detallan a continuación:

El modelo lineal mixto unicarácter para el análisis del peso al nacimiento se expresó como:

$$
P N C_{k l m n i j}=\mu+S C_{k}+N P_{l}+E_{m}+T N_{n}+b_{j}+a_{i}+\varepsilon_{k l m n i j}
$$

Variable respuesta: PNC

Variables predictoras:

- SC: efecto fijo sexo de la cría.

- NP: efecto fijo número de parto.

- E: efecto fijo estación.

- TN: efecto fijo total de nacidos.

- $a$ : efecto genético aditivo del animal (aleatorio) del i-ésimo animal.

- $b$ : efecto de la j-ésima camada (aleatorio).

Donde:

$$
\mathbf{a} \sim N\left(\mathbf{0}, A \sigma_{a}^{2}\right), \mathbf{b} \sim N\left(\mathbf{0}, \mathbf{I} \sigma_{b}^{2}\right) \text { y } \mathbf{a} \sim N\left(0, \mathbf{I} \sigma_{e}^{2}\right) .
$$

$A$ es la matriz de relaciones genéticas aditivas.

Los efectos aleatorios son independientes, es decir todas las covarianzas son ceros. $S_{a}^{2}, S_{b}^{2}$ y $S_{e}^{2}$ son la varianza aditiva, varianza respecto a la camada y varianza residual respectivamente.

El modelo lineal mixto unicarácter para el análisis del peso al destete se expresó como:

$P D C_{k l m n i j}=\mu+S C_{k}+N P_{l}+E_{m}+N V_{n}+E D C_{f}+b_{j}+a_{i}+\varepsilon_{k l m n f j}$

Variable respuesta: PDC

Variables predictoras:

- SC: efecto fijo sexo de la cría.

- NP: efecto fijo número de parto.

- E: efecto fijo estación.

- NV: efecto fijo total de nacidos vivos.

- EDC: efecto fijo edad de la cría al destete.

- $a$ : efecto genético aditivo del animal (aleatorio) del i-ésimo animal.

- $b$ : efecto de la j-ésima camada (aleatorio).

Donde:

$A$ es la matriz de relaciones genéticas aditivas. $\mathbf{a} \sim N\left(\mathbf{0}, A \sigma_{a}^{2}\right), \mathbf{b} \sim N\left(\mathbf{0}, \mathbf{I} \sigma_{b}^{2}\right)$ y $\stackrel{\mathbf{a}}{2} N\left(0, \mathbf{I} \sigma_{e}^{2}\right)$. Los efectos aleatorios son independientes, es decir todas las covarianzas son ceros. $\boldsymbol{S}_{a}^{2}, \boldsymbol{S}_{b}^{2}$ y $\boldsymbol{S}_{e}^{2}$ son la varianza aditiva, varianza respecto a la camada y varianza residual respectivamente.

En el campo del mejoramiento genético (animal o de plantas) es a menudo de interés comparar la magnitud de los diferentes componentes de varianza que contribuyen a la varianza total de la variable respuesta (o carácter en estudio). Por tanto la variación genética entre los individuos de un grupo pueden ser comparados con la cantidad de variación debido a causas no genéticas en una cantidad llamada heredabilidad $\left(h^{2}\right)$. El método para 
calcular la variabilidad depende de la estructura genética de la población considerada.

La estimación de la heredabilidad de un carácter en sentido estricto es la proporción de la variabilidad fenotípica observada (variable respuesta) que es de origen genético (Gutiérrez J.P., 2010), es decir:

$$
h^{2}=\frac{s_{a}^{2}}{s_{a}^{2}+s_{b}^{2}+s_{e}^{2}}
$$

\section{Resultados y discusión}

Las estimaciones de la heredabilidad utilizando la metodología REML fueron $0.158 \pm 0.036$ para el peso al nacimiento y $0.24 \pm 0.05$ para peso al destete como se muestra en la tabla 1, las cuales no fueron ligeramente diferentes a la que encontró Rodríguez (2013) quien realizó estimaciones bajo la misma metodología pero mediante un modelo multivariante tomando como respuestas las dos características y estimando la heredabilidad del peso al nacimiento en $0.17 \pm 0.05 \mathrm{y}$ del peso al destete en $0.26 \pm 0.07$. En general los valores estimados pueden ser considerados bajos. Sin embargo Solarte et al. (2002) con un modelo también multivariante estimó valores ligeramente más bajos para la heredabilidad del peso al nacimiento $(0.12 \pm 0.05)$ y peso al destete $(0.13 \pm 0.05)$ que con los obtenidos en este estudio.

Tabla 1. Estimados de los componentes de varianza y heredabilidad para peso al nacimiento y peso al destete.

\begin{tabular}{ccccccc}
\hline Carácter & $\sigma_{a}^{2}$ & $\sigma_{b}^{2}$ & $\sigma_{e}^{2}$ & $h^{2} \pm s . e$ & $c^{2} \pm$ s.e. & Log-L \\
\hline $\begin{array}{c}\text { Peso al } \\
\text { nacimiento }\end{array}$ & 118.3 & 342.44 & 289.74 & $0.158 \pm 0.036$ & $0.4563 \pm 0.02$ & -20477.08 \\
Peso al destete & 1026.98 & 2054.26 & 1110.2 & $0.24 \pm 0.05$ & $0.49 \pm 0.026$ & -16003.6 \\
\hline
\end{tabular}

$s_{a}^{2}, s_{b}^{2}, \sigma_{e}^{2}, c^{2}$ y $h^{2}$ : varianza aditiva, varianza respecto a la camada, varianza residual, proporción de la varianza ambiental materna y heredabilidad aditiva respectivamente

\section{Conclusiones}

Los estimados de heredabilidad obtenidas vía REML a través de modelos mixtos con una característica como variable respuesta fueron similares a los obtenidos por medio de un modelo multivariante estimado con la misma información. Al compararla con estudios anteriores en cuyes, la heredabilidad estimada para el peso al destete fue algo mayor que con el peso al nacimiento, pero ambos bajas por lo que se diría que la expresión fenotípica de estas características está también influenciada por factores diferentes a la acción aditiva.

Podría recomendase desde una perspectiva estadística realizar una validación de los supuestos del modelo, análisis de residuales que permita realizar una mejor selección de modelos en trabajos futuros.

\section{Agradecimientos}

Al personal de la granja de Cuyes de Cieneguilla de la Universidad Nacional Agraria La Molina por brindar las facilidades en la toma de datos.

\section{Literatura citada}

Gilmour, A. R.; Cullis, B. R.; Welham, S. J. and Thompson, R. (1999). ASREML, reference manual. Biometric bulletin, no 3, NSW Agriculture, Orange Agricultural. Institute, Forest Road, Orange 2800 NSW Australia.

Gutierrez, J. P. 2010. Iniciación a la valoración genética animal. Metodología adaptada al EEES. UCM Editorial Complutense. 355 p.
Mrode, R. 2014. Linear Model for the Prediction of Animal Breeding Value. 3 ed. CABI. Edinburgh UK. 343 p.

R Core Team. 2013. R: A language and environment for statistical computing. R Foundation for Statistical Computing, Vienna, Austria. URL http://www.R-project. $\underline{\text { org/ }}$

Rodríguez, H. 2013. Evaluación fenotípica y estimación de parámetros genéticos en características productivas y reproductivas en cuyes de la granja de Cieneguilla. Tesis doctoral en Ciencia Animal. UNALM. 79 p.

Solarte, C.; Imuez, A.M. y Pérez, T. 2002. Modelo animal multicarácter para la estimación de parámetros genéticos del Cavia porcellus en Colombia. Revista Cubana de Ciencia Agrícola, 36(1): 19-24

Solarte, C.; Soto, F. y Pérez, T. 2002. Modelo animal multicarácter para la selección de reproductores Cavia porcellus en Colombia. Revista Cubana de Ciencia Agrícola, 36(1): 25-29.

Thompson, R. 2008. Estimation of quantitative genetic parameters. Proc. R. Soc. B 275:679-686.

\section{Anexo}

Algunas sentencias en $\mathbf{R}$ para estimar componentes de varianza para peso al nacimiento.

library(asreml)

dat.ped $<$ - dat[,1:3]

datped.ainv $<$ - asreml.Ainverse(dat.ped)\$ginv

$\mathrm{fm}<-$ asreml $(\mathrm{PNC} \sim \mathrm{SC}+\mathrm{NP}+\mathrm{E}+\mathrm{TN}$, random $=$

$\sim \mathrm{CA}+$ ped $(\mathrm{ID}, \mathrm{var}=\mathrm{T})$, ginverse $=\operatorname{list}(\mathrm{ID}=$ datped.

ainv), data $=$ dat) 